\title{
Análise dos casos de nefrite lúpica na cidade de Catanduva - SP
}

\author{
Analysis of lúpus nefhritis cases in Catanduva - SP \\ Análisis de los casos de nefrite lúpica en la ciudad de Catanduva - SP
}

Francieli Carolini Pasqualetto ${ }^{1 \star}$, Bruno Henrique Estareli ${ }^{1}$ Nathália Maciel Maniezzo Stuchi².

\section{RESUMO}

Objetivo: Avaliar o número de casos de nefrite lúpica em pacientes com Lúpus Eritematoso Sistêmico (LES) na cidade de Catanduva-SP entre os anos de 2010 a 2015. Métodos: A análise dos prontuários, obtidos nos hospitais Emílio Carlos e Padre Albino da cidade de Catanduva-SP, resultou em um total de 30 pacientes com LES. Resultados: Todos os pacientes selecionados eram do sexo feminino e o maior número de casos foi na faixa etária de 30 a 39 anos (33,3\%), além de haver aumento no número de atendimentos devido à doença ao longo dos anos. A ocorrência de casos de comprometimento renal decorrente de nefrite lúpica ocorreu em 7 desses pacientes (23.3\%). Todos os pacientes com nefrite lúpica apresentavam fator antinuclear reagente e proteinúria $24 \mathrm{~h}$ aumentada, destes, 5 apresentaram hipertensão arterial sistêmica. Esses fatores, juntamente com anormalidades no sedimento urinário são determinantes em uma avaliação do comprometimento renal, sendo a biópsia o principal padrão para se determinar a cronicidade da doença. Conclusão: Uma maior elucidação da nefrite lúpica nesses pacientes não foi possível devido à falta de informações a respeito da biópsia nos prontuários, impossibilitando assim a classificação dos mesmos, além disso ocorreu erros na notificação da patologia, tornando um diagnóstico impreciso.

Palavras-chave: Lúpus Eritematoso Sistêmico, Nefrite Lúpica, Glomerulonefrite.

\begin{abstract}
Objective: To evaluate the number of notification cases in patients with Systemic Lupus Erythematosus (SLE) in the city of Catanduva-SP between the years 2010 and 2015. Methods: Data analysis at Hospitals Emílio Carlos and Padre Albino in the city of Catanduva-SP, in a total of 30 patients with SLE. Results: All patients were females and the highest number of cases was between 30 and 39 years old (33.3\%), in addition to an increase in the number of visits due to long-term illness. Absence of cases of renal impairment due to lupus nephritis in 7 patients (23.3\%). All patients with lupus nephritis had reactive antinuclear factor and increased $24 \mathrm{~h}$ proteinuria, of which 5 had systemic arterial hypertension. These factors, together with abnormalities in the urinary sediment are determinant in an evaluation of renal impairment, a biopsy being the main standard for determining a chronicity of the disease. Conclusion: The increased elucidation of lupus nephritis was not possible due to a lack of information about the biopsy in the medical records, making it impossible to assimilate them as patients were submitted to pathology, making the diagnosis imprecise.
\end{abstract}

Keywords: Systemic Lupus Erythematosus, Lupus Nephritis, Glomerulonephritis.

1 Universidade Estadual de Campinas (UNICAMP), Campinas - SP. * E-mail: f.pasqualetto@hotmail.com.

2 Centro Universitário Padre Albino (UNIFIPA), Catanduva - SP.

${ }^{3}$ Universidade Estadual Paulista (UNESP-IBILCE); Instituto Adolfo Lutz (IAL), São José do Rio Preto - SP; Centro Universitário Padre Albino (UNIFIPA), Catanduva - SP. 


\section{RESUMEN}

Objetivo: Evaluar el número de casos de nefritis lúpica en pacientes com Systemic Lupus Erythematosus (LES) en la ciudad de Catanduva-SP entre los años 2010 a 2015. Métodos: El análisis de los prontuarios, obtenidos en los hospitales Emilio Carlos y Padre Albino de la ciudad de Catanduva-SP, resultó en un total de 30 pacientes con LES. Resultados: Todos los pacientes seleccionados eran del sexo femenino y el mayor número de casos fue en el grupo de edad de 30 a 39 años (33,3\%), además de haber aumento en el número de atendimientos debido a la enfermedad a lo largo de los años. La ocurrencia de casos de compromiso renal derivada de nefritis lúpica ocurrió en 7 de esos pacientes (23.3\%). Todos los pacientes con nefritis lúpica presentaban factor antinuclear reactivo y proteinuria $24 \mathrm{~h}$ aumentada, de éstos, 5 presentaron hipertensión arterial sistémica. Estos factores, junto con anormalidades en el sedimento urinario, son determinantes en una evaluación del compromiso renal, siendo la biopsia el principal estándar para determinar la cronicidad de la enfermedad. Conclusión: Una mayor elucidación de la nefritis lúpica en estos pacientes no fue posible debido a la falta de informaciones acerca de la biopsia en los prontuarios, imposibilitando así la clasificación de los mismos, además ocurrió errores en la notificación de la patología, haciendo un diagnóstico impreciso.

Palabras claves: Systemic Lupus Erythematosus, Lupus Nefritis, Glomerulonephritis.

\section{INTRODUÇÃO}

Até o ano de 1872, o lúpus era considerado apenas como doença cutânea, mas nesse mesmo ano, Kaposi (KAPOSI, 1872), deu ênfase em sua natureza sistêmica.

A prevalência do Lúpus Eritematoso Sistêmico (LES) varia de 15-50/100.000 habitantes e os fatores genéticos e ambientais são determinantes para o desenvolvimento da enfermidade. Ocorre em sua maioria em mulheres, em uma proporção de 8:1 a 13:1 em relação aos homens, a ocorrência em adultos também é maior em comparação com as outras faixas etárias (COSTALLAT, 1995), além disso, é mais incidente em hispânicos e afro-americanos, do que em caucasianos. Dentre os fatores ambientais, se enquadram a relação de substâncias como a procainamida, luz ultravioleta, estresse, infecções e fatores étnicos e socioeconômicos.

Para o diagnóstico de LES, considera-se, no mínimo, a ocorrência de quatro critérios clínicos e laboratoriais, entre as onze alterações determinadas pelo American College of Rheumatology (TAN et al. 1982), sendo eles: rash malar, lesão discóide, fotossensibilidade, úlceras da mucosa oral, artrite nãodeformante, serosite, doença renal, envolvimento do sistema nervoso central, alterações hematológicas, alterações imunológicas e fator antinúcleo.

O Lúpus se divide basicamente em dois tipos, o cutâneo (ou discóide) e o sistêmico, sendo que o primeiro é uma doença exclusiva da pele e o segundo apresenta comprometimento de outros órgãos, como os rins, pulmões e coração. Ainda assim, essas duas classificações não são totalmente delimitadas, embora mais raramente, cerca de $1 \%$ a $5 \%$ dos casos de Lúpus Eritematoso Discóide podem evoluir para a forma sistêmica da doença (LAHITA, 1987).

A prevalência de comprometimento renal aparece em $60 \%$ dos casos de lúpus e é um fator determinante na morbimortalidade, sendo um marcador de prognóstico, uma vez que pode levar à insuficiência renal, hipertensão arterial e síndrome nefrótica (HOUSSIAU, 2004). Em pacientes com problemas renais é encontrado desde proteinúria assintomática, até casos mais severos de insuficiência renal irreversível. $\mathrm{O}$ comprometimento renal pode ser apenas anatômico, com a presença de alterações histológicas e ausentes de sintomas clínicos, porém entre 50 e $70 \%$ dos afetados apresentam quadro de nefropatia clínica (MOREIRA e CARVALHO, 2001). Dentre as manifestações clínicas, destaca-se o surgimento de baixo volume urinário, hipertensão arterial, surgimento de edemas. Já entre as manifestações laboratoriais, salienta-se o aumento das taxas séricas de uréia e creatinina, alterações em proteinúria de 24 horas, redução do clearence de creatinina, alterações no sedimento urinário, aumento dos títulos dos anticorpos anti-DNAn, redução da taxa de complemento, diminuição dos níveis plaquetários, entre outros (MOREIRA e CARVALHO, 2001 e APPEL et al. 1978). 
Para se fazer a detecção do comprometimento renal (nefropatia ou nefrite lúpica), o método mais recomendado segundo a Organização Mundial da Saúde (OMS), é a biópsia, que continua sendo o padrão ouro para informar o grau e cronicidade da doença, sendo importante para uma orientação terapêutica (MOREIRA e CARVALHO, 2001 e APPEL et al. 1978). A classificação da OMS é feita de acordo com os achados anatomopatológicos nos exames de microscopia óptica, eletrônica e de fluorescência. A classe I e II aplica-se a glomerulonefrite mínima e proliferativa mesangial, respectivamente. Classe III e IV indica glomerulonefrite segmentar focal e difusa com lesões necrosantes, respectivamente. A classe $V$ aplica-se a glomerulonefrite membranosa e a classe VI indica esclerose avançada (GALINDO-IZQUIERDO et al. 2016).

Há 30 anos, somente cerca de $50 \%$ dos pacientes sobreviviam por mais de dois anos após o diagnóstico de LES (MOREIRA e CARVALHO, 2001). Na atualidade, essa porcentagem subiu para $90 \%$ e em $80 \%$ dos casos, as pessoas acometidas por lúpus sobrevivem por mais 10 anos, $65 \%$ conseguem uma sobrevida de 20 anos depois do diagnóstico (MOREIRA e CARVALHO, 2001).

O Lúpus Eritematoso Sistêmico (LES) é uma doença inflamatória crônica, autoimune e multissistêmica, apresentando ampla variedade de manifestações, que envolvem diferentes órgãos (CALTIK et al. 2013). As manifestações clínicas são mediadas por imunocomplexos que ocasionam danos teciduais, que são ocasionados por anticorpos patogênicos, imunocomplexos, linfócitos $\mathrm{T}$ e auto-anticorpos, porém esta interação e a etiologia da doença, ainda não são totalmente conhecidas.

Os antígenos que são liberados por apoptose, são responsáveis por produzirem anticorpos auto-reativos, sendo um importante fator ambiental na sensibilização dos portadores à exposição solar e à luz ultravioleta (ABBAS et al. 2008).

A produção de anticorpo auto-reativos acontece por meio do reconhecimento de fragmentos apoptóticos pela lgM, receptor de Célula $\mathrm{B}(\mathrm{BCR})$ que vai reconhecer os fragmentos, processá-los e apresentá-los ao linfócito T auxiliar através do MHC II (Complexo principal de histocompatibilidade de classe 2). Ao reconhecer o antígeno, o linfócito produzirá citocinas, ativando o linfócito $B$ que se modificará em plasmócito, secretando anticorpos auto reativos com especificidade para o DNA, originando imunocomplexos através dessa ligação antígeno anticorpo (CARROLL, 2004).

Segundo pesquisadores, estes imunocomplexos são depositados nos glomérulos renais, articulações, pele e vasos sanguíneos os complexos imunes que não foram excluídos da circulação e sendo capaz de gerar desencadeamento de resposta inflamatória no local (NAIRN e HELBERT, 2004).

Devido à dificuldade em se diagnosticar o LES e por consequência relatar os casos de nefrite lúpica correlacionados à doença, o presente estudo teve como objetivo avaliar o número de casos de nefrite lúpica na cidade de Catanduva-SP entre os anos de 2010 a 2015.

\section{MÉTODOS}

Este trabalho foi aprovado pelo comitê de Ética e Pesquisa do Centro Universitário Padre Albino sob o número (CAAE) 58031516.9.0000.5430, e seguiu as recomendações da Resolução do Conselho Nacional de Saúde 196/96. Os casos de nefrite lúpica foram determinados de acordo com as informações obtidas de prontuários dos Hospitais Emílio Carlos e Padre Albino de Catanduva-SP, foram avaliados os casos entre os anos de 2010 a 2015. Foram coletadas todas as informações obtidas por meio dos prontuários, como por exemplo, sexo, idade, etnia, estado civil, ano do diagnóstico, medicamentos utilizados pelo paciente, dentre outras informações relevantes quanto a evolução da doença contidas nos laudos. Os dados encontrados foram discutidos de acordo com a literatura científica pertinente ao assunto, sendo obtidos por meio de acesso ao Pubmed, Scielo, Lilacs, sites do ministério da saúde, OMS (Organização Mundial de Saúde), e livros publicados na área.

\section{RESULTADOS}

Após análise dos prontuários, obtidos nos Hospitais Padre Albino e Emílio Carlos na cidade de CatanduvaSP, encontrou-se um total de 30 pacientes que apresentavam a Classificação Internacional de Doenças (CID)

REAS/EJC | Vol.11(1) | DOI: https://doi.org/10.25248/reas28_2019 Página 2365 de 2368 
referente ao Lúpus, os dados se referem aos anos de 2010 a 2015. Foi possível observar que todos os pacientes selecionados eram do sexo feminino e a faixa etária na qual prevaleceu o número de casos foi de 30 a 39 anos $(33,3 \%)$.

O número de casos por ano aumentou significativamente entre 2010 e 2015, sendo que no ano de 2014 , 10 casos (33,3\%) foram registrados nos hospitais, enquanto em 2010 e 2011 foi de apenas 1 caso por ano $(3,3 \%)$.

Quanto a Classificação Internacional de Doenças (CID), observou-se 5 diferentes tipos que apresentam relação com a patologia estudada (Tabela 1): L930 (Lúpus Eritematoso Discóide); L391 (Lúpus Eritematoso Cutâneo Subagudo); L932 (Outras formas de Lúpus Eritematoso Localizado); M328 (Outras formas de Lúpus Eritematoso Sistêmico); M329 (Lúpus Eritematoso Sistêmico não especificado). Sendo o CID L930 o mais frequente, com 19 ocorrências (63,3\%).

Foi observado a ocorrência de casos de comprometimento renal decorrente de nefrite lúpica em 7 desses pacientes $(23.3 \%)$.

Tabela 1 - Características dos pacientes portadores de Lúpus avaliados no período de 2010 a 2015.

\begin{tabular}{|c|c|c|c|c|c|c|}
\hline Casos & Ano & Sexo & Raça & Idade & CID & Nefrite Lúpica \\
\hline Caso 1 & 2010 & Fem. & Branca & 12 & L932 & Ausente \\
\hline Caso 2 & 2011 & Fem. & $\mathrm{NI}$ & 34 & L930 & Ausente \\
\hline Caso 3 & 2012 & Fem. & Branca & 54 & L930 & Presente \\
\hline Caso 4 & 2012 & Fem. & Branca & 49 & L930 & Ausente \\
\hline Caso 5 & 2012 & Fem. & $\mathrm{NI}$ & 52 & L930 & Ausente \\
\hline Caso 6 & 2012 & Fem. & Branca & 37 & L930 & Ausente \\
\hline Caso 7 & 2013 & Fem. & $\mathrm{NI}$ & 55 & L932 & Ausente \\
\hline Caso 8 & 2013 & Fem. & Branca & 29 & L930 & Ausente \\
\hline Caso 9 & 2013 & Fem. & Branca & 48 & L930 & Ausente \\
\hline Caso10 & 2013 & Fem. & $\mathrm{NI}$ & 35 & L930 & Presente \\
\hline Caso 11 & 2013 & Fem. & $\mathrm{NI}$ & 15 & L930 & Ausente \\
\hline Caso 12 & 2014 & Fem. & Branca & 31 & L932 & Ausente \\
\hline Caso 13 & 2014 & Fem. & $\mathrm{NI}$ & 37 & L930 & Ausente \\
\hline Caso 14 & 2014 & Fem. & Branca & 43 & L930 & Presente \\
\hline Caso 15 & 2014 & Fem. & Branca & 21 & L930 & Ausente \\
\hline Caso 16 & 2014 & Fem. & Parda & 14 & L932 & Ausente \\
\hline Caso 17 & 2014 & Fem. & Negra & 39 & L932 & Presente \\
\hline Caso 18 & 2014 & Fem. & $\mathrm{NI}$ & 35 & L932 & Ausente \\
\hline Caso 19 & 2014 & Fem. & Branca & 52 & L932 & Ausente \\
\hline Caso 20 & 2014 & Fem. & Branca & 31 & L931 & Ausente \\
\hline Caso 21 & 2014 & Fem. & Branca & 20 & L931 & Presente \\
\hline Caso 22 & 2015 & Fem. & Branca & 27 & M329 & Ausente \\
\hline Caso 23 & 2015 & Fem. & Branca & 46 & L930 & Ausente \\
\hline Caso 24 & 2015 & Fem. & $\mathrm{NI}$ & 42 & L930 & Ausente \\
\hline Caso 25 & 2015 & Fem. & Negra & 25 & L930 & Presente \\
\hline Caso 26 & 2015 & Fem. & Negra & 39 & L930 & Ausente \\
\hline Caso 27 & 2015 & Fem. & Branca & 45 & L930 & Ausente \\
\hline Caso 28 & 2015 & Fem. & Branca & 18 & L930 & Ausente \\
\hline Caso 29 & 2015 & Fem. & Parda & 35 & M328 & Ausente \\
\hline Caso 30 & 2015 & Fem. & $\mathrm{NI}$ & 45 & M328 & Presente \\
\hline
\end{tabular}

Legenda: $\mathrm{NI}=$ Não informado

REAS/EJC | Vol.11(1) | DOI: https://doi.org/10.25248/reas28_2019 Página 2366 de 2368 


\section{DISCUSSÃO}

Segundo Lourenço et al., (2004), a nefrite lúpica é uma das principais causas de morbidade e mortalidade em pacientes com Lúpus Eritematoso Sistêmico, segundo Appel et al., (2004) aproximadamente 60\% dos indivíduos afetados desenvolverão algum comprometimento renal no curso da doença (APPEL et al. 2007), desta forma o reconhecimento e tratamento rápido da doença renal são vitais para uma melhor resposta a terapia utilizada (BERTSSIAS et al. 2008).

Dentre os 30 pacientes identificados neste estudo, 7 deles (23,3\%) apresentavam nefrite lúpica. Dados a respeito de incidência e prevalência de lúpus e suas comorbidades variam bastante de um país para o outro, até mesmo dentre regiões de um mesmo país (DANCHENKO et al. 2006). O que contribui para essa diferença é a seleção de pacientes, fatores étnicos, sociais e ambientais, além dos critérios usados para a classificação da doença. Alguns pesquisadores encontraram valores semelhantes aos obtidos neste trabalho. Um estudo de Cervera et al., (2003) envolvendo sete países europeus, durante 10 anos, encontrou 279 pacientes (27.9\%) com nefrite ativa em um total de 1000 pacientes estudados (CERVERA et al. 2003). Segundo Eilertsen et al., (2011), encontraram a nefrite lúpica em $26 \%$ dos pacientes na Noruega, e Rúa-Figueroa et al., (2014), verificou 30\% de nefrite histológica confirmada em pacientes com Lúpus na Espanha, entre os anos de 2011 e 2012. (EILERTSEN et al. 2011 e RÚA-FIGUEROA et al. 2014)

Maiores incidências de nefrite lúpica também são encontradas na literatura, como por exemplo, em estudo realizado por Hiraki (2012) com crianças americanas entre 2000 e 2004, onde 1106 (37\%) crianças estudadas foram identificadas com a doença (HIRAKI, 2012).

Entretanto, uma pesquisa dinamarquesa Hermansen et al., (2016), durante os anos de 1995 e 2001 observou que 233 (14\%) em um total de 1644 indivíduos lúpicos apresentavam nefrite durante o progresso da doença (HERMANSEN et al. 2016).

De acordo com a literatura, Mak et al. (2007) a grande maioria dos casos de nefrite associada ao LES acomete mulheres com menos de 55 anos, assim como a proporção notada neste trabalho sendo a média de idade de 37 anos no grupo de pacientes com nefrite (MAK et al. 2007).

Os dados clínicos como proteinúria, hipertensão arterial e anormalidades no sedimento urinário são critérios chave para a determinação do comprometimento renal em pacientes com lúpus. Todos os casos aqui analisados apresentavam valores aumentados de proteinúria 24h. Em uma revisão da literatura de Cameron (1999) foi relatado que $100 \%$ dos pacientes tiveram aumento de proteinúria (CAMERON, 1999).

No presente trabalho, cinco dentre os sete pacientes apresentaram hipertensão arterial sistêmica (HAS), e em todos os sete casos foi detectado positivamente o fator antinuclear (FAN), que segundo o estudo de Caltik et al., (2013) é detectado em mais de $95 \%$ dos pacientes com LES, enquanto o exame de anti-DNA foi reagente em 4 deles (CALTIK et al. 2013).

Dois casos apresentaram pielonefrite, sendo que um deles apresentou insuficiência renal aguda, representando $14,3 \%$ do total de pacientes com nefrite lúpica. Hermansen et al., (2016) evidenciaram que $8,9 \%$ das pessoas com nefrite lúpica desenvolvem insuficiência renal aguda por ano na Dinamarca (HERMANSEN et al. 2016). Em nossos achados podemos destacar um caso de óbito decorrente de insuficiência renal aguda em um paciente com 45 anos, branco, com piora do caso relatado nos laudos, que além de apresentar os sinais característicos de glomerulonefrite evolui para anemia hemolítica.

Não foi possível explanar sobre a nefrite lúpica nos pacientes estudados devido à falta de informações a respeito de biópsia nos prontuários, impossibilitando assim a classificação dos mesmos.

Por meio dos dados coletados foi possível observar que ainda nos dias atuais o diagnóstico, classificação da doença e o acompanhamento de pacientes com Lúpus são muito controversos, como exemplo podemos citar o fato de que 4 dos 7 pacientes com nefrite lúpica foram classificados de acordo com o CID L930, que se refere ao Lúpus Eritematoso Discóide, e sabe-se que a maioria das manifestações dos pacientes geralmente ocorre por lesões na pele (GRONHAGEN et al. 2011), sendo improvável um paciente evoluir de Lúpus Eritematoso Discóide para Lúpus Eritematoso Sistêmico com possível comprometimento renal (Merola

REAS/EJC | Vol.11(1) | DOI: https://doi.org/10.25248/reas28_2019 Página 2367 de 2368 
et al 2013 e Santiago-Casas et al. 2012). Tais achados reafirmam a necessidade de atualização dos profissionais da saúde em relação a estas enfermidades, já que o acompanhamento devido da doença pode prevenir complicações durante a evolução da mesma.

\section{CONCLUSÃO}

A ocorrência de nefrite lúpica em pacientes portadores de Lúpus apresentou aumento progressivo no número de atendimentos decorrentes à doença durante os anos avaliados, demonstrando a necessidade de atualização dos profissionais da saúde sobre o tema e monitoramento contínuo dos pacientes, verificando corretamente os sintomas para que no momento de diagnosticar o paciente isto ocorra de maneira adequada para que obtenha uma maior qualidade na definição do prognostico e no possível tratamento, evitando equívocos no momento do tratamento da doença.

\section{REFERÊNCIAS}

1. ABBAS AK, LICHTMAN AH, PILLAI S. Imunologia celular e molecular. Elsevier, 6aㅡ edição, 2008, p.420-438.

2. APPEL GB, SILVA FG, PIRANI CL, et al. Renal involvement in systemic lupus erythematosus (SLE): a study of 56 patients emphasizing histologic classification. Medicine. 1978; 57(5):371-410.

3. APPEL GB, RADHAKRISHNAN J, D'AGATI V. Secondary glomerular disease. In The Kidney. 8th edition. Philadelphia, PA: Saunders;2007:1067-1148.

4. BERTSIAS G, GORDON C, BOUMPAS DT. Clinical trials in SLE: lessons learned from the past as we proceed to the future - the EULAR recommend ations for the management of SLE and the use of end-points in clinical trials.Lupus 2008, 17:437-442.

5. CALTIK A, DEMIRCIN G, BULBUL M, et al. An unusual case of ANA negative systemic lupus erythematosus presented with vasculitis, long-standing serositis and full-house nephropathy. Rheumatol Int, 33:219-22.

6. CARROLL MC. A protective role for innate immunity in systemic lupus erythematosus, Nature Review Immunologys, v.4, p.825-831, 2004.

7. CAMERON JS. Lupus nephritis. J Am SocNephrol 1999, 10:413-424.

8. CERVERA R, KHAMASHTA MA, FONT J, et al. Morbidity and mortality in systemic lupus erythematosus during a 10-year period: a comparison ofearly and late manifestations in a cohort of 1,000 patients. Medicine, 82(5):299-308.

9. COSTALLAT LTL. Lúpus eritematoso sistêmico. Medical Master: Anais de Atualização Médica, 1995; 121-134.

10. DANCHENKO N, SATIA JA, ANTHONY MS. Epidemiology of systemic lupus erythematosus: a comparison of worldwide disease burden. Lupus 2006; 15:308-18.

11. EILERTSEN GO, FISMEN S, HANSSEN TA, et al. Decreased incidence of lupus nephritis in northern Norway is linked to increased use of antihypertensive and anticoagulant therapy. Nephrol Dial Transplant 2011; 26:620-627.

12. GALINDO-IZQUIERDO M, RODRIGUEZ-ALMARAZ E, PEGO-REIGOSA JM, et al. Characterization of Patients With Lupus Nephritis Included in a Large Cohort From the Spanish Society of Rheumatology Registry of Patients With Systemic Lupus Erythematosus (RELESSER). 2016, Medicine (Baltimore), 2010, 15:482-490.

13. GRONHAGEN CM, FORED CM, GRANATH F, et al. Cutaneous lupus erythematosus and the association with systemic lupus erythematosus: a population-based cohort of 1088 patients in Sweden. Br J Dermatol, 164 (2011),1335-1341.

14. HIRAKI LT, FELDMAN CH, LIU J, et al. Prevalence, incidence, and demographics of systemic lupus erythematosus and lupus nephritis from 2000 to 2004 among children in the US Medicaid beneficiary population. Arthritis Rheum 2012; 64:2669-76.

15. HOUSSIAU FA. Management of lupus nephritis: an update. J Am Soc Nephrol, 2004;15:2694-2704.

16. KAPOSI M. New beitagezurkenntnis des lúpus erythematosus. Arch Dermatol Spyph, 1872; 4:36. 8.

17. LAHITA RG. In systemic lupus erythematosus, 2nd ed. New York (NY): Churchill Livingstone; 1987:620.

18. MAK A, MOK CC, CHU WP, et al. Renal damage in systemic lupus erythematosus: a comparative analysis of different age groups. Lupus 2007, 16:28-34.

19. MARIE-LOUISE F, HERMANSEN ML, Lindhardsen J, et al. Incidence of Systemic Lupus Erythematosus and Lupus Nephritis in Denmark: A Nationwide Cohort Study. J Rheumatol, 2016, 43(7): 1335-1339.

20. MEROLA JF, PRYSTOWSKY SD, IVERSSEN C, et al. Association of discoid lupus erythematosus with other clinical manifestations among patients with systemic lupus erythematosus. J Am Acad Dermatol 2013; 69: 19-24.

21. MOREIRA C, CARVALHO MAP. Reumatologia: Diagnóstico e tratamento. 2nd Ed. Brasil: MEDSI Editora Médica e Científica Ltda; 2001. p.423-47.

22. NAIRN R, HELBERT M. Imunologia para estudantes de medicina, Gunabara Koogan, 1a edição, 2004, p.241-242; 253-259.

23. RÚA-FIGUEROA I, LÓPEZ-LONGO FJ, CALVO-ALÉN J, et al. Registro nacional de pacientes com lupus eritematoso sistémico de La Sociedad Española de Reumatología: objetivos y metodología. Reumatol Clin. 2014; 10:17-24.

24. SANTIAGO-CASAS Y, VILA LM, MCGWIN G, et al. Association of discoid lupus erythematosus with clinical manifestations and damage accrual in a multiethnic lupus cohort. Arthritis Care Res (Hoboken) 2012; 64: 704-712.

25. TAN EM, COHEN AS, FRIES JF et al. The 1982 revised criteria for the classification of systemic lupus erythematosus. Arthritis Rheum, $1982 ; 25: 1271-7$.

REAS/EJC | Vol.11(1) | DOI: https://doi.org/10.25248/reas28_2019 Página 2368 de 2368 